\title{
Derivação de Casos de Testes Funcionais: uma Abordagem Baseada em Modelos UML*
}

\author{
Alex Orozco $^{1}$, Kleinner Oliveira ${ }^{2}$, Flávio Oliveira ${ }^{1}$, Avelino Zorzo ${ }^{1}$ \\ ${ }^{1}$ Departamento de Informática - Pontifícia Universidade Católica do Rio Grande do Sul \\ Avenida Ipiranga, 6681 - 90619-900 - Porto Alegre - RS - Brasil \\ ${ }^{2}$ Departamento de Informática - Pontifícia Universidade Católica do Rio de Janeiro \\ R. Marquês de São Vicente, 225 RDC - 22.453-900- RJ - Brasil \\ \{alex.orozco,flavio.oliveira, zorzo\} @pucrs.br, \\ kfariaseinf.puc-rio.br
}

\begin{abstract}
In this paper we present a model-based testing approach that aims at identifying, automating and deriving functional test cases in whole or in part from UML models that describe some aspects of the system under test. Our approach provide a considerable reduction of the effort on the test generation, increasing the effectiveness of the tests, shortening the testing cycle, and avoiding tedious and error-prone editing of a suite of hand-crafted tests. Finally, we present a case study to demonstrate the practicality and usefulness of the proposed approach.
\end{abstract}

Resumo. Neste artigo apresentamos uma abordagem de teste de software baseada em modelos que foca na identificação, automatização e derivação completa, ou parcial, de casos de teste a partir da composição de modelos UML que descrevem alguns aspectos do sistema que está sendo testado. Nossa abordagem provê uma considerável redução de esforço na geração de testes, aumentando a eficiência dos testes, diminuindo o ciclo de teste, e evitando a confecção tediosa e propensa a erros de um conjunto de casos de testes. Por fim, apresentamos um estudo de caso para demonstrar de modo prático os benefícios da abordagem proposta.

\section{Introdução}

Um fator significante que evidencia a dificuldade encontrada durante o processo de desenvolvimento de software é a existência de um gap conceitual entre o domínio do problema e o domínio da solução [France 2006] [France 2007]. Diferentes paradigmas de desenvolvimento têm sido propostos com o objetivo de solucionar este gap, tais como: desenvolvimento de software orientado a objetos (OOP), desenvolvimento de software orientado a aspectos (AOP), e recentemente o desenvolvimento de software dirigido por modelos (MDD).

Estes paradigmas de desenvolvimento são utilizados em algum processo de desenvolvimento de software (por exemplo, RUP (tradicional) [Kruchten 1999] e SCRUM (ágil) [Schwaber 2004]) durante a execução das atividades inerentes ao processo. Porém, independente do paradigma de desenvolvimento e do tipo de processo utilizado, precisa-se, sem dúvida, da elaboração de produtos de software de qualidade. Logo, as atividades e técnicas relacionadas a testes de software têm se diversificado e

* Este trabalho foi desenvolvido em colaboração com a HP Brasil P\&D. 
vêm ganhando cada vez mais ênfases dentro dos processos de desenvolvimento visando atender a esta necessidade. Um exemplo destas técnicas seria o teste de software dirigido por modelos. Se por um lado fica claro que a realização de testes tem um papel importante na aferição da qualidade de um software, por outro lado, a execução incompleta ou inadequada de testes pode proporcionar problemas que possivelmente comprometerão o bom funcionamento, o desempenho e a confiança no funcionamento do sistema.

Diante do dinamismo e da complexidade dos sistemas de software atuais, tornase extremamente desafiante para os testadores criar, derivar casos de testes e colocar teste de software em prática dado o problema em mãos. Consequentemente, testar software exige extensiva habilidade dos testadores tanto em relação aos diferentes tipos de testes quanto às técnicas de automatização e aplicação de teste "antecipado", ou seja, nas fases inicias do processo. Isto pode causar o surgimento de complexidades adicionais, o que torna a atividade de teste mais custosa e difícil. A automação dos testes pode reduzir o esforço requerido para as atividades de testes de software. Através da automação, os testes podem ser realizados em um menor tempo, ao invés de demorarem horas para serem executados manualmente, podendo alcançar uma diminuição de esforço em mais de $80 \%$ [Fewster e Graham 1999]. A aplicação de teste automatizado nas organizações pode reduzir gastos ou esforços de forma indireta. Além disso, a automação permite produzir softwares de melhor qualidade mais rapidamente do que seria possível através de testes manuais.

Neste artigo apresentamos uma abordagem de teste de software baseada em modelos que foca na identificação, automatização e derivação completa, ou parcial, de casos de teste funcional a partir do diagrama de atividades da UML (Unified Modeling Language) [OMG 2007]. O objetivo é realizar teste funcional nas fases iniciais do processo de desenvolvimento. Nossa abordagem provê uma considerável redução de esforço na geração de testes através da composição dos diferentes diagramas de atividades do sistema a ser testado e um único diagrama, evitando assim atividades redundantes e conseqüentemente casos de teste redundantes. É apresentado um estudo de caso para demonstrar de modo prático os benefícios da abordagem proposta.

Este artigo é organizado da seguinte forma. A Seção 2 discorre sobre o referencial teórico desta pesquisa. A Seção 3 e Seção 4 apresentam a abordagem proposta. A Seção 5 apresenta um estudo de caso que a mostrando a abordagem na prática. A Seção 6 apresenta os trabalhos relacionados. Finalmente, na Seção 7, são apresentadas as conclusões e os trabalhos futuros.

\section{Referencial Teórico}

Nesta seção serão discutidos os principais conceitos necessários para a compreensão da técnica de automatização de teste proposta apresentada neste artigo.

\subsection{Teste Funcional}

Teste funcional consiste na realização de um conjunto de atividade que visam localizar discrepâncias entre o comportamento do sistema sobre teste e as especificações de comportamento esperado. Tais especificações são descrições precisa do comportamento do programa do ponto de vista do usuário final. Em outras palavras, o teste funcional tenta verificar as funcionalidades do sistema considerando uma dada entrada de dados, 
processamento e resposta. Para prover um teste funcional, as especificações são analisadas para derivarem um conjunto de casos de testes. Exceto quando utilizado em pequenos programas, o teste funcional é uma atividade conhecida como caixa preta, ou seja, se confia que um processo de teste unitário anterior alcançou um critério de cobertura lógica aceitável.

\subsection{UML}

A UML [OMG 2007] trata-se de uma linguagem padrão para modelagem de sistemas orientados a objetos, sendo usada tanto na academia quanto na indústria. Apresenta um conjunto de diagramas que são usados para representar aspectos estáticos e dinâmicos de um sistema em desenvolvimento. Dentre estes diagramas, encontra-se o diagrama de atividades que, na sua essência, representa os fluxos conduzidos por algum tipo de processamento. Trata-se de um gráfico de fluxo, mostrando o fluxo de controle de uma atividade para outra. Neste artigo, este diagrama é um elemento central para permitir a derivação automática dos casos de teste funcional.

\subsection{Máquina de Estados Finitos com Entradas e Saídas}

Uma Máquina de Estados Finitos (MEF) com entradas e saídas é definido como uma 6tupla $(S, I, A, R, \triangle, T)$, onde $S$ é o conjunto finito de estados, $I \subset S$, é conjunto de estados iniciais, $A$ é o alfabeto finito de símbolos de entrada e $R$ é o conjunto de possíveis saídas ou respostas. O conjunto $\Delta \subset S \times A$ é o domínio da relação de transição $T$, que é uma função de $\Delta$ para $S \times R$. A relação de transição descreve como a máquina reage ao receber entrada $a \in A$ quando $s \in S$, assumindo que $(s, a) \in \Delta$. Interpreta-se $(s, a) \notin \Delta$ como: o símbolo de entrada não pode ser aceito no determinado estado. Quando $T(s, a)=(s$ ', $r)$, o sistema move-se para o novo estado $s^{\prime}$ e responde como saída $r$. Se $T$ não é uma função, mas mais exatamente uma relação que associa cada par de estado de entrada com um conjunto não vazio de pares de estados de saída, é dito que o autômato finito é não determinístico, e é interpretado como o conjunto de possíveis respostas para um estímulo de entrada em um certo estado [Friedman et al 2002].

\subsection{Método UIO}

O método UIO (Unique Input/Output) é um método para a geração de um conjunto de sequiências de entrada para testar uma MEF [Delamaro et al 2007]. Para a geração destas sequiências, este método utiliza-se de seqüências UIO. As sequiências UIO são utilizadas para verificar se a MEF está em um determinado estado em particular. Sendo assim, cada estado da MEF poderá possuir uma seqüência UIO distinta.

Desta forma, como afirma Delamaro et al (2007), "para cada transição de um estado $s_{i}$ para $s_{j}, f_{\mathrm{s}}\left(s_{i}, x\right)$, com algum $x$, é definida uma sequiência que conduz do estado inicial a $s_{i}$, aplica-se o símbolo de entrada e, em seguida, aplica-se a sequiência UIO do estado que deveria ser atingido. Sendo assim, cada sequiência é da forma $P\left(s_{i}\right) \cdot x \cdot$ $\mathrm{UIO}\left(s_{j}\right)$, sendo que $P\left(s_{i}\right)$ é uma sequiência que leva a MEF do estado inicial ao estado $s_{i}$ e $\mathrm{UIO}\left(s_{j}\right)$ é uma seqüência para $s_{j}$ ”. 


\section{Derivação de Casos de Testes Funcionais}

Para possibilitar a geração de casos de testes, este trabalho propõe a utilização de diagrama atividades dos sistemas que está sendo testado. Através deste modelo, é possível, ainda na fase de requisitos, começar a pensar em como o plano de teste deve ser criado. Porém, o diagrama de atividades não contém informações suficientes para a geração dos casos de teste. Com isso, é necessário inserir as características relacionadas a teste funcional. A Figura 1 exibe o overview da abordagem proposta.

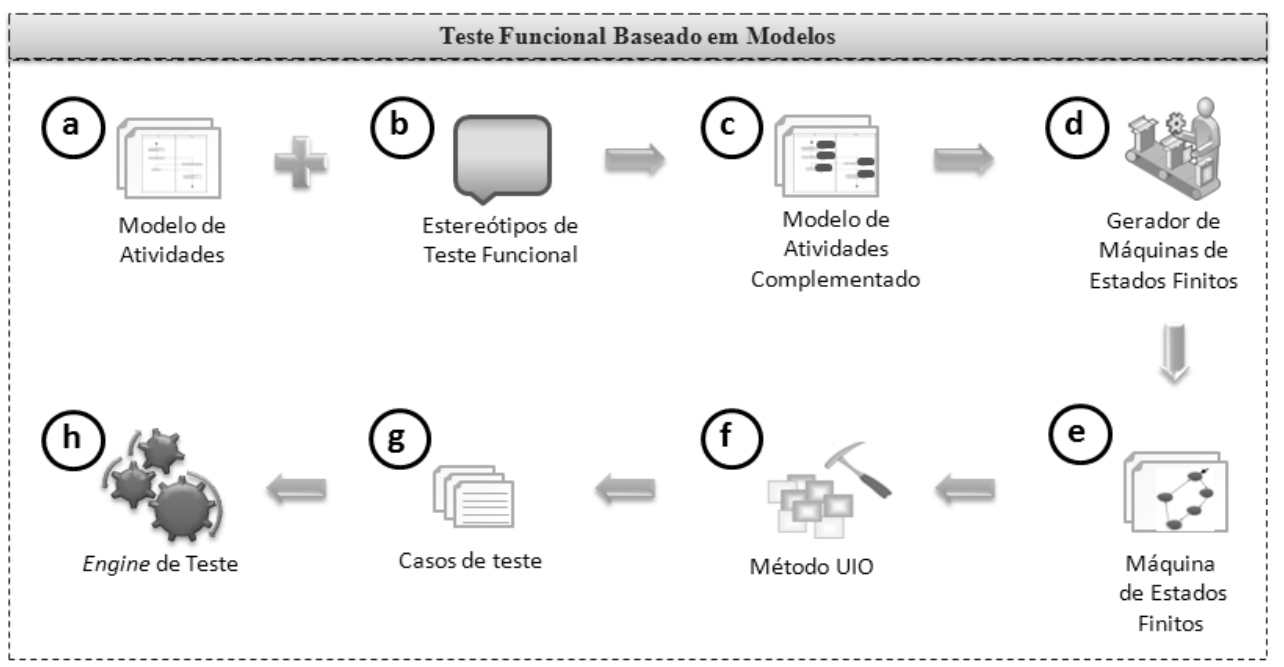

Figura 1. Um overview da abordagem proposta.

De posse dos modelos de atividades do sistema em teste (a), é inserido em cada atividade relevante para o teste um estereótipo definido como $\langle$ FTStep $\rangle$ (b). Este estereótipo é composto de duas marcações, uma denominada FTAction, responsável por especificar a ação a ser realizada no sistema, e a outra denominada FTExpectResult, responsável por especificar o resultado esperado da ação definida na FTAction.

Após a inclusão destas informações, o modelo de atividades complementado (c) é convertido em uma máquina de estados finitos com entrada e saída (d), onde o alfabeto de entrada é formado pelo conteúdo das marcações FTAction, o alfabeto de saída é formado pelo conteúdo das marcações FTExpectedResult e os estados são formados pelas atividades do diagrama de atividades. Considerando um diagrama de atividades composto por duas atividades $A_{1}$ e $A_{2}$, onde $A_{1}$ leva a $A_{2}$, a máquina de estados finitos equivalente será formada pelos estados $A_{1}$ e $A_{2}$, a entrada da transição $\mathrm{A}_{1} \rightarrow \mathrm{A}_{2}$ será o conteúdo da marcação FTAction da atividade $\mathrm{A}_{2}$ e a saída da transição será o conteúdo da marcação FTExpectedResult da atividade $\mathrm{A}_{2}$.

Nesta máquina de estados finitos (e) é aplicado o método de geração de sequiência de entrada para testar a máquina conhecido como método UIO (f) [Delamaro et al 2007]. Este método gera uma seqüência única de entrada e saída para alcançar cada estado da máquina de estados finitos. Desta forma, cada seqüência UIO resulta em um caso de teste (g). Com isto, ainda durante a fase de requisitos é possível ter uma idéia sobre os prováveis casos de teste a serem realizados quando a aplicação estiver implementada, sendo estes casos de teste refinados à medida que o processo de desenvolvimento evolui, bastando atualizar os dados existentes nas marcações do 
modelo e gerar os casos de teste novamente. Esta abordagem resulta na vantagem que para cada atividade exposta no diagrama de atividades é gerado um caso de teste, provendo um critério de cobertura bastante amplo.

Quando a aplicação estiver implementada, o modelo de atividades é atualizado com as informações relevantes para automatizar a geração de scripts de teste, sendo inseridas nas marcações do estereótipo $<$ FTStep $>$, em linguagem de script, a ação que o objeto da interface da aplicação representada pela atividade do modelo deve realizar e o seu respectivo resultado esperado. Desta forma, os casos de teste gerados com estas informações podem ser executados em uma engine de teste (h), automatizando o processo de teste.

\section{Especialização da Abordagem Proposta}

A abordagem descrita na Seção 3 tenta proporcionar a derivação de casos de testes funcionais a partir do diagrama de atividades do sistema em teste, sendo necessário que cada diagrama de atividades seja exposto aos passos descritos. Para sistemas simples, esta abordagem é bastante eficiente, mas para sistemas de um porte expressivo começa a apresentar deficiências.

Supondo um sistema com três diagramas de atividades, onde cada diagrama possui quatro atividades em sequiência e os três diagramas possuem uma atividade denominada Inserir nome de usuário. Ao expor estes diagramas à abordagem da seção 3 , cada atividade resulta em um caso de teste. Sendo assim, como resultados existirão doze casos de teste. Se a atividade Inserir nome de usuário for idêntica para os três diagramas, a abordagem gerou três casos de teste redundantes, um caso de teste para cada vez que a atividade foi exposta nos diagramas. Se situações como esta existirem em sistemas com um grande volume de diagramas de atividades, a quantidade de casos de teste redundantes será de um número elevado.

Para solucionar esta deficiência, é proposta a realização da composição de todos os diagramas de atividades em um único diagrama, como exibido na Figura 2.

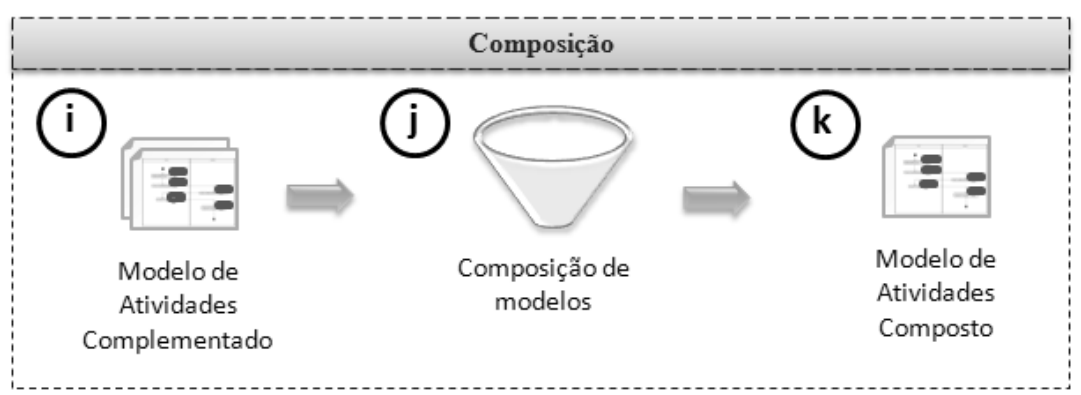

Figura 2. Processo de composição dos diagramas de atividades.

A composição pode ser definida como uma atividade transformação, onde dois modelos $M_{a}$ e $M_{b}$ são transformado em $M_{a b}$, o resultado da composição. Em nossa abordagem $M_{a}$ e $M_{b}$ são diagramas de atividades. Desse modo, para realizar a composição, é preciso definir quando as atividades do diagrama são consideradas iguais. Neste trabalho foi definido que duas atividades são iguais quando a ação a ser realizada na atividade (conteúdo da marcação FTAction) e o resultado esperado desta ação (conteúdo da marcação FTExpectedResult) são iguais. 
Desta forma, aplica-se a todos os diagramas de atividades do sistema em teste (i) esta regra de composição (j), resultando em um único modelo de atividades (k). Com isso eliminam-se as atividades redundantes do ponto de vista do teste funcional. Com esta solução, a abordagem apresentada na Figura 1 é atualizada para a exibida na Figura 3 , onde o passo (c) é substituído pelo processo da Figura 2, sendo os modelos de atividades (a) com o estereótipo inserido (b) passam pelo processo de composição (c) e o modelo resultante da composição é convertido em uma máquina de estados finitos (d).

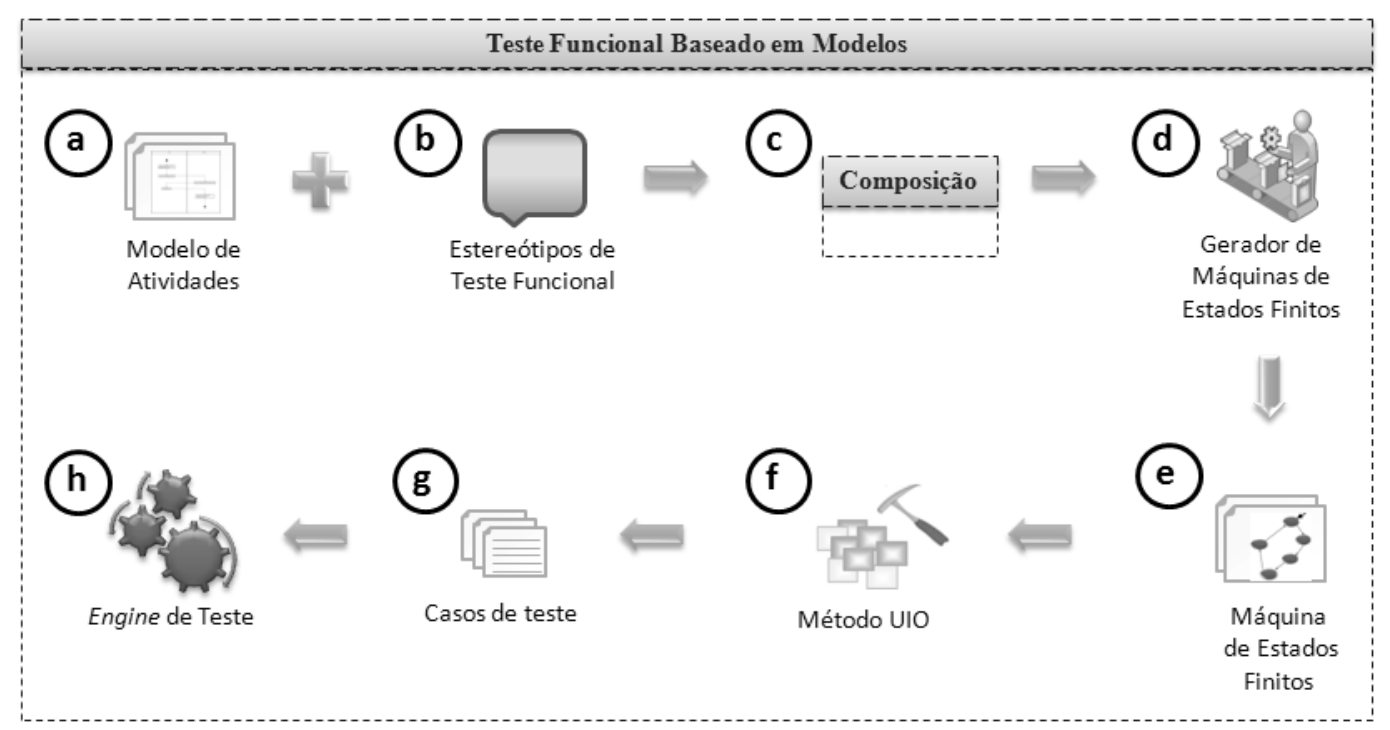

Figure 3. Abordagem completa.

\section{Estudo de caso}

Para avaliar a abordagem proposta, foi definido e desenvolvido um estudo de caso que é descrito nesta seção. Este estudo é concentrado nas funcionalidades presentes em calculadores tradicionais, especificamente as funcionalidades apresentadas pelas operações de Soma e Divisão. Basicamente, são definidos dois casos de uso, Somar e Dividir. Os respectivos diagramas de atividades para estes dois casos de uso são exibidos na Figura 4.

Analisando as atividades dos diagramas para aplicar a composição, verifica-se que somente a primeira atividade dos dois diagramas são consideradas iguais, baseado na regra de composição que para duas atividades serem iguais, o conteúdo das marcações FTAction e FTExpectedResult precisam ser iguais. Nas atividades de selecionar a operação, embora o resultado esperado seja o mesmo, a ação é diferente. $\mathrm{O}$ mesmo ocorre com a atividade Calcular, embora ambas possuam o mesmo conteúdo em FTAction, o resultado esperado é diferente. 


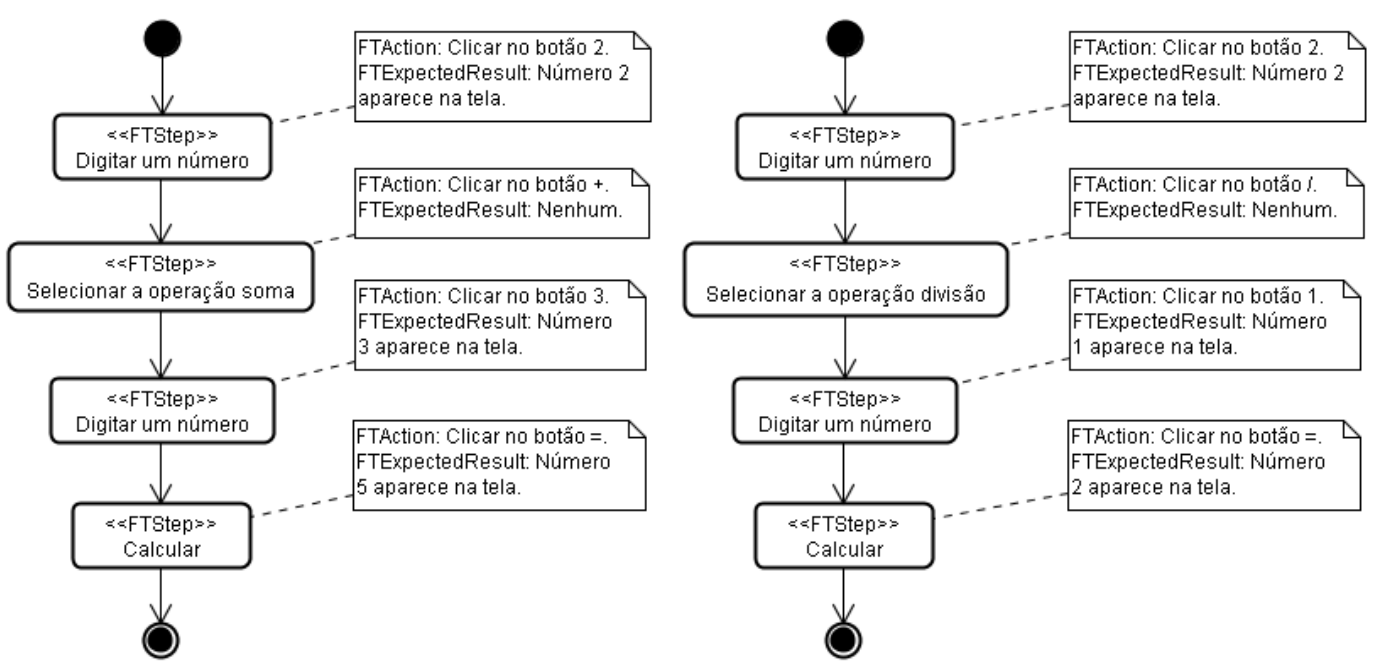

Figura 4. Diagramas de atividades referentes aos casos de uso Somar e Dividir.

O diagrama resultante da composição é exibido na Figura 5.

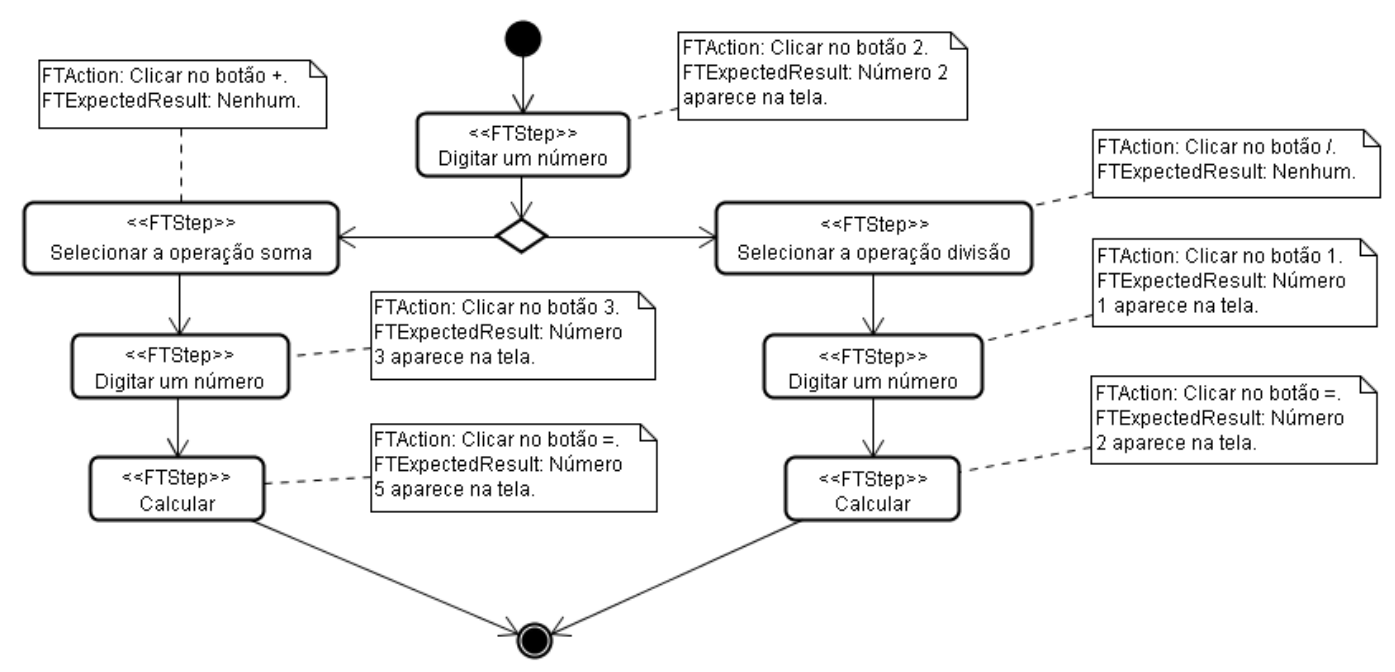

Figura 5. Diagrama de atividades após a composição.

Após a composição, este diagrama resultante é inserido em um gerador de máquinas de estados finitos com entrada e saída, onde a máquina de estados finitos resultante é exibida na Figura 6. 


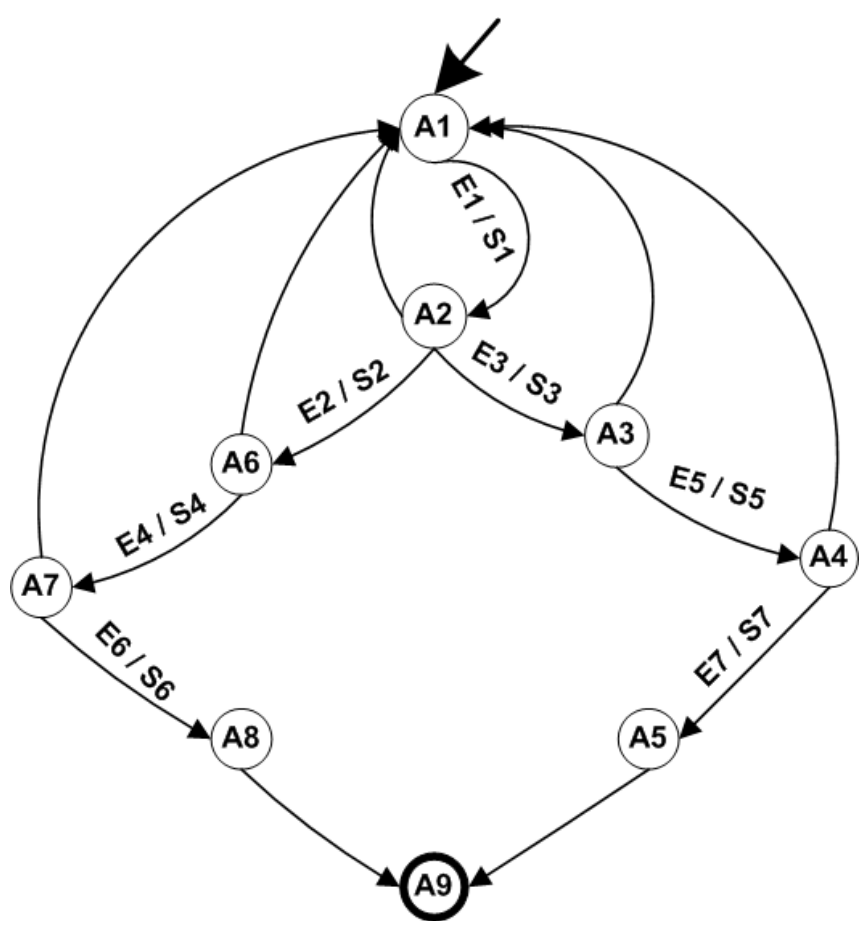

\section{LEGENDA}

E1 - Clicar no botão 2

S1 - Número 2 aparece na tela

E2 - Clicar no botão +.

S2 - Nulo.

E3 - Clicar no botão /.

S3 - Nulo

E4 - Clicar no botão 3.

S4 - Número 3 aparece na tela

E5 - Clicar no botão 1.

S5 - Número 1 aparece na tela.

E6 - Clicar no botão $=$.

S6 - Número 5 aparece na tela.

E7 - Clicar no botão $=$.

S7 - Número 2 aparece na tela.

Figura 6. Máquina de estados finitos equivalente.

Aplicando o método UIO nesta máquina de estados finitos, o resultado será $\{(\mathrm{E} 1 / \mathrm{S} 1),(\mathrm{E} 1 / \mathrm{S} 1, \mathrm{E} 2 / \mathrm{S} 2),(\mathrm{E} 1 / \mathrm{S} 1, \mathrm{E} 2 / \mathrm{S} 2, \mathrm{E} 4 / \mathrm{S} 4),(\mathrm{E} 1 / \mathrm{S} 1, \mathrm{E} 2 / \mathrm{S} 2, \mathrm{E} 4 / \mathrm{S} 4, \mathrm{E} 6 / \mathrm{S} 6)$, (E1/S1, E3/S3), (E1/S1, E3/S3, E5/S5), (E1/S1, E3/S3, E5/S5, E7/S7)\}. Desta forma, cada sequiência UIO resulta em um caso de teste. Para a seqüência (E1/S1, E2/S2, E4/S4, E6/S6) a descrição do caso de teste resultante seria:

1. Clicar no botão 2.

Resultado Esperado: Número 2 aparece na tela.

2. Clicar no botão + .

3. Clicar no botão 3 .

Resultado Esperado: Número 3 aparece na tela.

4. Clicar no botão $=$.

Resultado Esperado: Número 5 aparece na tela.

Para a execução dos casos de teste de forma automatizada, foi utilizada a ferramenta de teste funcional HP Quick Test Professional (QTP) [QTP 2008], por ser uma ferramenta para automação de testes funcionais largamente adotada pela indústria. Para a utilização desta ferramenta, foram substituídos, nos diagramas de atividades dos casos de uso Somar e Dividir, os conteúdos das marcações FTAction e FTExpectedResult por comandos do QTP. A abordagem aqui proposta não se limita apenas para aplicação no QTP, podendo ser utilizada em qualquer ferramenta de 
automação de testes funcionais baseadas em scripts, onde os comandos da ferramenta são inseridos da mesma forma que este trabalho exemplifica para o QTP.

A Figura 7 exemplifica o diagrama de atividades do caso de uso Somar dotado dos comandos do QTP.

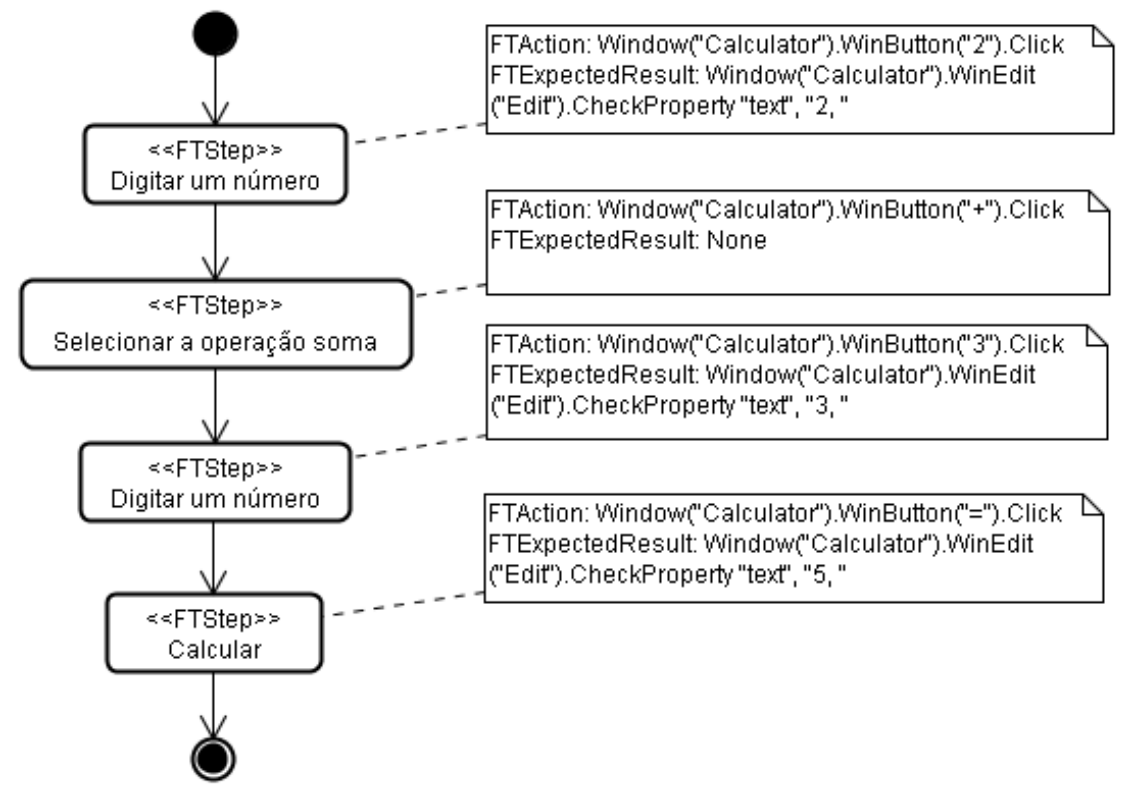

Figura 7. Diagrama de atividades referente aos casos de uso Somar, dotado de comandos da ferramenta Quick Test Professional.

Após estes diagramas serem compostos, ser gerada a máquina de estados finitos equivalente e aplicado o método UIO, o resultado do mesmo caso de teste descrito anteriormente, agora no formato de script para o QTP, é apresentado abaixo:

Window("Calculator").WinButton("2").Click

Window("Calculator").WinEdit("Edit").CheckProperty "text", "2, "

Window("Calculator").WinButton("+").Click

Window("Calculator").WinButton("3").Click

Window("Calculator").WinEdit("Edit").CheckProperty "text", "3, "

Window("Calculator").WinButton("=").Click

Window("Calculator").WinEdit("Edit").CheckProperty "text", "5, "

Cabe ressaltar que neste estudo de caso foi necessário determinar o valor a ser inserido para a realização da soma, pois um número é representado na calculadora como um objeto botão e não como um campo de texto, ou seja, está sendo testada a funcionalidade do botão 2 da calculadora, e não somente o valor 2 para a realização da soma.

\section{Trabalhos relacionados}

Alguns trabalhos apontam tentativas de facilitar o processo de testes derivando os casos de teste a partir de modelos. Oliveira et al (2007) propõe a derivação de casos de teste 
de desempenho a partir de diagramas de atividades e casos de uso. Tais diagramas são complementados com estereótipos da UML Profile for Schedulability, Performance and Time [OMG 2008], onde os dados existentes nos diagramas mais os estereótipos provêem informações suficientes para a geração de uma rede de Petri estocástica generalizada [Marsan et al 1984], podendo assim simular as características de desempenho tanto de forma determinística quanto estocástica.

Vários outros trabalhos abordam o tema, tais como testes funcionais a partir de modelos de máquinas de estados finitos estendidas [Pretschner et al 2005], testes de integração a partir de diagramas UML de estados (StateChart Diagrams) [Hartmann et al 2000]. Neto (2007) analisa setenta e oito trabalhos sobre testes baseados em modelos, sendo quarenta e sete deles baseados em modelos UML. Rodrigues (2008) utiliza testes de desempenho baseados em modelos UML para auxiliar na realocação de recursos em ambientes virtualizados. Peralta (2008) especifica e gera casos de testes de segurança baseados em modelos UML.

Um diferencial da abordagem aqui proposta em relação aos demais trabalhos é o uso de mecanismos de composição de modelos com o objetivo de diminuir redundâncias na geração de casos de teste, visto que freqüentemente uma atividade encontra-se em diferentes diagramas de atividades do sistema a ser testado, fazendo com que ao utilizar estes diagramas individualmente para a geração de casos de teste, esta mesma atividade se repita, resultando em casos de teste redundantes.

\section{Conclusões e trabalhos futuros}

Se os testes são vistos como elementos centrais para a garantia de qualidade de um produto de software, então um processo de desenvolvimento de software deve naturalmente se preocupar com como e quando estes testes são realizados. A fim de serem usados de uma forma mais eficiente, tais testes devem ser executados de uma forma automatizada e preferencialmente aplicada nas primeiras etapas do processo de desenvolvimento.

Com isso, foi apresentada uma proposta de derivação de casos de testes funcionais a partir de modelos de atividades da UML. Para realizar esta derivação, foi definida uma abordagem baseada na inserção de informações relevantes aos testes funcionais através da aplicação de estereótipos. Após isto, é realizada a composição dos diferentes modelos de atividades do sistema sob teste em um único modelo, o modelo composto. A partir deste modelo composto, é gerada uma máquina de estados finitos com entradas e saídas. Nesta máquina é aplicado um algoritmo para a criação de sequiências de entradas e saídas que são convertidas em casos de teste para o sistema sob teste.

Foi implementada uma ferramenta que possibilitou colocar a abordagem em prática e realizar um estudo de caso. A avaliação inicial da abordagem tem demonstrado a aplicabilidade e a viabilidade da técnica desenvolvida. Obviamente, mais investigações sobre sua eficiência, quanto ao seu uso na derivação de casos de testes com diagramas de atividades da UML de tamanho elevado, são necessárias. Neste sentido, os trabalhos futuros serão concentrados em projetar e executar avaliações da abordagem através de testes na indústria. Pretende-se refinar o mecanismo de composição de modelos, visando diminuir ainda mais as redundâncias na geração dos casos de teste. 


\section{Referências}

Delamaro, M., Maldonado, J. e Jino, M. (2007) . "Introdução ao teste de software". Rio de Janeiro: Campus.

Fewster, M. e Graham, D. (1999) "Software test automation: effective use of test execution tools". New York, NY, USA: ACM Press/Addison-Wesley Publishing Co.

France, R. e Rumpe, B. (2007) "Model-Driven Development of Complex Software: A Research Roadmap," in Future of Software Engineering (FOSE'07) co-located with ICSE'07, Minnesota, EUA, p. 37-54.

France, R., Ghosh, S. e Dinh-Trong, T. (2006) "Model Driven Development Using UML 2.0: Promises and Pitfalls," IEEE Computer Society, v. 39, n. 2, p. 59-66.

Friedman, G. et al. (2002) "Projected state machine coverage for software testing", In: International symposium on software testing and analysis. New York, NY, USA: ACM, p.134-143.

Hartmann, J., Imoberdorf, C. e Meisinger, M. (2000) "UML-based integration testing". In: International symposium on software testing and analysis, New York, NY, USA: ACM. p. 60-70.

HP Quick Test Professional - QTP (2008). Disponível em <https://h10078.www1.hp. $\mathrm{com} / \mathrm{cda} / \mathrm{hpms} / \mathrm{display} / \mathrm{main} / \mathrm{hpms} \_c o n t e n t . j s p ? \mathrm{zn}=\mathrm{bto} \& \mathrm{cp}=1-11-12724 \% 5 \mathrm{E} 1352 \_40$ 00_100__. Acesso em: 17 nov. 2008.

Kruchten, P. (1999). "The Rational Unified Process: an introduction”. Addison-Wesley Longman Publishing Co., Inc., Boston, MA, USA.

Marsan, M., Conte, G. e Balbo, G. (1984) "A class of generalized stochastic petri nets for the performance evaluation of multiprocessor systems". ACM Transactions on Computer Systems, ACM, New York, NY, USA, v. 2, n. 2, p. 93-122.

Mcgregor, J. (2001) “Testing a Software Product Line”. Technical Report, CMU/SEI 2001-TR- 022, Software Engineering Institute, Carnegie Mellon University.

Neto, A. et al. (2007) "A survey on model-based testing approaches: a systematic review". In: International workshop on empirical assessment of software engineering languages and technologies. New York, NY, USA: ACM Press. p. 31-36.

Pretschner, A. et al. (2005) "One evaluation of model-based testing and its automation". In: International conference on software engineering. Saint Louis, MO, USA: ACM Press. p. 392-401.

Oliveira, F. et al. (2007) "Performance testing from UML models with resource descriptions". In: Brazilian workshop on systematic and automated software testing. João Pessoa, PB, Brazil: Brazilian Computer Society. p. 47-54.

Object Management Group - OMG (2008). "UML Profile for Schedulability, Performance and Time". Disponível em <http://www.omg.org/technology/ documents/formal/schedulability.htm>. Acesso em: 17 nov. 2008.

Object Management Group - OMG (2007). "Unified Modeling Language: Infrastructure version 2.1". Disponível em < http://www.omg.org/docs/formal/07-0206.pdf >. Acesso em: 17 nov. 2008. 
Schwaber, K. (2004). "Agile Project Management With Scrum". Microsoft Press, Redmond, WA, USA.

Rodrigues, E. "Alocação de recursos em ambientes virtualizados" Dissertação (Mestrado em Ciência da Computação). Faculdade de Informática, Pontifícia Universidade Católica do Rio Grande do Sul, Porto Alegre, 2008.

Peralta, K. "Uma Estratégia para Especificação e Geração de Casos de Teste de Segurança usando Modelos UML" Dissertação (Mestrado em Ciência da Computação). Faculdade de Informática, Pontifícia Universidade Católica do Rio Grande do Sul, Porto Alegre, 2008. 